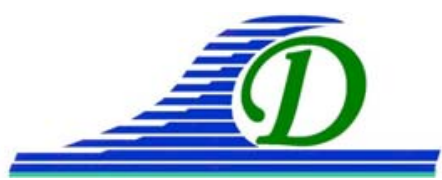
XIII ${ }^{\text {èmes }}$ Journées Nationales Génie Côtier - Génie Civil
Dunkerque, 2-4 juillet 2014

DOI:10.5150/jngcgc.2014.080 (c) Editions Paralia CFL

disponible en ligne - http://www.paralia.fr - available online

\title{
Méthode des domaines fictifs appliquée à l'interaction d'un solide mobile avec un écoulement à surface libre en vue de l'application aux systèmes houlomoteurs
}

\author{
Benoît DUCASSOU ${ }^{1}$, Stéphane ABADIE ${ }^{1}$, Marcela CRUCHAGA ${ }^{2}$
}

1. Université de Pau et des Pays de l'Adour, Laboratoire SIAME EA 4581, 1 allée du parc Montaury, 64600 Anglet, France.

benoit.ducassou@univ-pau.fr ; stephane.abadie@univ-pau.fr

2. Universidad de Santaigo de Chile, Departamento de Ingeniería Mecánica, Av Lib Bdo. O'Higgins, 3363 Santiago de Chile, Chile.

marcela.cruchaga@usach.cl

\section{Résumé :}

Ce travail présente une méthode numérique originale pour l'interaction fluide/structure basée uniquement sur la simulation numérique des équations de Navier-Stokes avec un suivi d'interface de type VOF. À l'aide d'une phase fluide de viscosité infinie, on cherche à simuler une structure solide indéformable à un seul degré de liberté en translation ou en rotation, et ce, de manière totalement implicite. Une force de rappel (proportionnelle au déplacement) ainsi qu'un amortissement (proportionnel à la vitesse de déplacement) peuvent être ajoutés sous la forme d'une force volumique dans la phase "solide". Des résultats préliminaires obtenus à l'aide de cette méthode sont présentés dans cet article.

Mots-clés : Navier-Stokes, VOF, Domaines fictifs, Pénalisation, Couplage fluide/structure, Houlomoteur.

\section{Introduction}

L'interaction entre un solide mobile et un fluide à surface libre est une thématique récurrente et commune à divers domaines. Dans ce travail nous proposons une méthode de simulation numérique originale basée sur les domaines fictifs (GLOWINSKI et al., 1999) pour étudier ce couplage. Le principe de cette méthode est de remplacer un système d'équation écrit dans un domaine à géométrie complexe (typiquement une forme complexe irrégulière) par un système d'équation "semblable", écrit dans un domaine à géométrie plus simple (typiquement un rectangle en 2D). L'objectif de notre travail est de simuler l'interaction entre une structure animée d'un mouvement simple (e.g., translation et rotation, voire éventuellement à plus long terme flexion) soumise à des forces extérieures de type rappel et amortissement et un fluide à surface libre. Les applications visées sont l'étude de systèmes de récupération de l'énergie de la houle et l'étude d'un système d'atténuation du ballottement. Une partie des développements réalisés dans ce travail de thèse trouve son cadre dans le projet national EMACOP. 


\section{Présentation de la méthode}

\subsection{Le modèle THETIS}

Le code THETIS, développé à l'Institut de Mécanique et d'Ingénierie de Bordeaux est utilisé dans cette étude pour résoudre les équations de Navier-Stokes incompressible suivantes :

$\nabla \cdot u=0$

$\rho\left(\frac{\partial u}{\partial t}+(u \cdot \nabla) u\right)=\rho g-\nabla p+\nabla \cdot\left[\mu\left(\nabla u+\nabla^{t} u\right)\right]$

Ce code permet de simuler un écoulement constitué de plusieurs phases non miscibles en suivant le déplacement de l'interface à l'aide d'une méthode de type VOF (ici VOFPLIC ; WILLIAM \& DOUGLAS, 1998). La discrétisation temporelle des équations de Navier-Stokes est faite en utilisant un schéma de GEAR d'ordre 1. La discrétisation spatiale est réalisée à l'aide des volumes finis (PATANKAR, 1990), le découplage Vitesse/Pression par la méthode du Lagrangien Augmenté (FORTIN \& GLOWINSKI, 1982).

\subsection{Couplage fluide/structure}

La méthode que nous proposons est tout d'abord basée sur la possibilité de rigidifier certaines zones du domaine fluide dans le modèle numérique. Pour cela, nous utilisons une technique de pénalisation basée sur la viscosité (RITZ \& CALTAGIRONE, 1999). Il suffit en effet d'imposer dans la zone que l'on cherche à rigidifier une valeur de viscosité très forte pour satisfaire numériquement un critère de non déformation. Supposons que le système d'équations (1) soit résolu par un algorithme quelconque qui converge, si $\mu$ est très grand, les contraintes visqueuses deviennent le terme prépondérant de l'équation de quantité de mouvement (système (1), seconde équation) dans la zone concernée du domaine fluide. Numériquement, le tenseur des vitesses de déformation qui vient en multiplication de $\mu$ doit tendre vers zéro pour assurer que la contrainte de cisaillement reste bornée, cela entraîne la "rigidification" de la zone concernée. En même temps, les termes d'inertie et de gravité existent toujours dans l'équation, permettant la simulation d'un solide rigide soumis à la gravité et en mouvement. Quant au terme de gradient pression, il est nul dans le solide indéformable. Il est en revanche non nul à l'interface de ce dernier et permet de transmettre les efforts de surface du fluide sur le solide qui sont capitaux pour décrire l'interaction fluide/structure. Différents auteurs ont utilisé cette technique avec le code THETIS. Ainsi, ABADIE et al. (2010), ont simulé le mouvement d'un bloc soumis à la gravité glissant dans l'eau sur une pente et ont montré que cette méthode permettait une estimation précise des efforts de traînée. De même, la déformation de la surface libre 


\section{XIII ${ }^{\text {èmes }}$ Journées Nationales Génie Côtier - Génie Civil \\ Dunkerque, 2-4 juillet 2014}

générée par la chute d'un bloc a été simulée avec succès grâce à cette approche dans la même référence.

Nous avons ensuite complété cette méthode en introduisant des forces extérieures de rappel (MOKRANI, 2012) et d'amortissement visqueux. Ces dernières interviennent dans les équations de Navier-Stokes sous la forme d'une force volumique $F_{v}$ (cf. équation (2)) qui ne sont actives que dans la zone fluide "rigidifiée" à laquelle on veut appliquer ce rappel ou cet amortissement :

$\rho\left(\frac{\partial u}{\partial t}+(u \cdot \nabla) u\right)=\rho\left(g+F_{v}\right)-\nabla p+\nabla \cdot\left[\mu\left(\nabla u+\nabla^{t} u\right)\right]$

Un des intérêts de cette méthode est que l'équilibre d'un solide soumis à des efforts de pression et forces extérieures classiquement ponctuelles (i.e., rappel et amortissement) est ici résolu par la même équation que celle qui régit l'écoulement externe.

\section{Premiers résultats numériques}

Nous présentons ici des résultats concernant le critère de rigidification et le cas d'un bloc en translation soumis à des efforts de rappel et d'amortissement.

\subsection{Critère de rigidité}

Pour pouvoir quantifier la déformation de la partie fluide que nous souhaitons rendre solide, nous avons défini un coefficient de déformation globale $C_{\text {tot }}$ comme suit :

$C_{\text {tot }}=\int C_{\text {def }}(t) d t=\sum_{i} C_{\text {def }}\left(t_{i}\right) \Delta t_{i}$

où $C_{\text {def }}(\mathrm{t})$ est la valeur maximale sur le bloc du coefficient de déformation locale (écrit ici en 2D):

$\operatorname{Cdef}_{i, j}=\sqrt{\varepsilon_{I i, j}^{2}+\varepsilon_{I I i, j}^{2}}$

avec $\varepsilon_{I i, j}, \varepsilon_{I I i, j}$ déformations principales en chaque point du maillage à un instant $\mathrm{t}$ donné.

$C_{\text {tot }}$ est un nombre majorant la déformation totale de la zone rigidifiée durant la simulation. En effet nous sommons ici des déformations comme si elles étaient toutes des allongements (i.e. >0) au même point alors que dans la réalité, ces déformations peuvent se compenser et concerner des zones différentes. Nous considérons ensuite la "rigidification" comme effective lorsque $C_{\text {tot }}<0,1 \%$, ce qui signifie une variation relative équivalente (au sens de ce qui vient d'être dit) de la longueur de l'objet $\Delta L / L<10^{-3}$.

Une série de simulations a permis de valider la méthode sur un problème de type poutre console modélisée à l'aide d'une phase fluide de masse volumique $\rho$ fixée arbitrairement à $1000 \mathrm{~kg} \mathrm{~m}^{-3}$. Nous avons étudié sa déformation sous l'effet de la gravité en faisant varier la viscosité du fluide (figure 1). Nous avons pu ainsi, dans ce cas particulier, déterminer la valeur optimale de la viscosité (i.e., $\mu_{o p t}=5 \times 10^{7}$ Pa s) permettant à la fois 


\section{Thème 5 - Énergies et ressources marines}

de rigidifier de manière satisfaisante la phase fluide (i.e., en respectant le critère $C_{\text {tot }}<0,1 \%$ ) tout en obtenant la convergence de l'algorithme de résolution de NavierStokes (figure 2, pas d'écoulement visible).

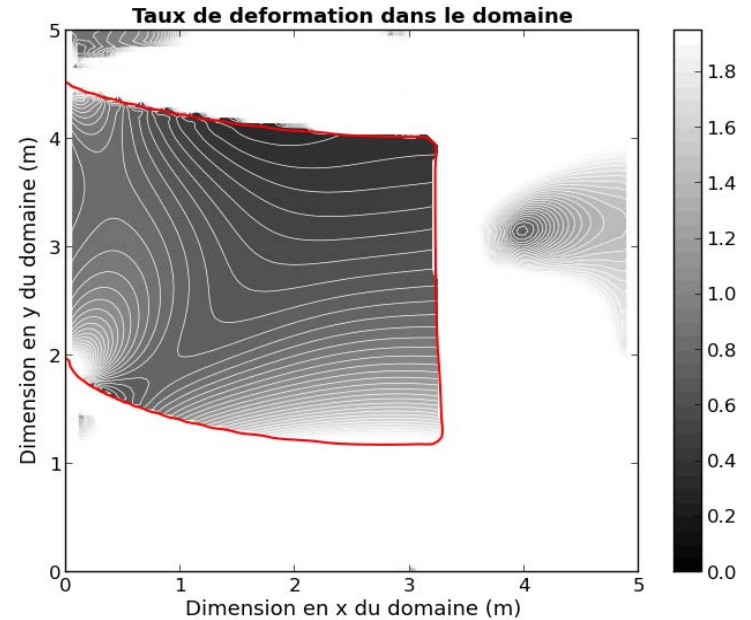

Figure 1. Déformée de la poutre à $t=0.38 \mathrm{~s}$ avec $\mu=1 \times 10^{4} \mathrm{Pas}$

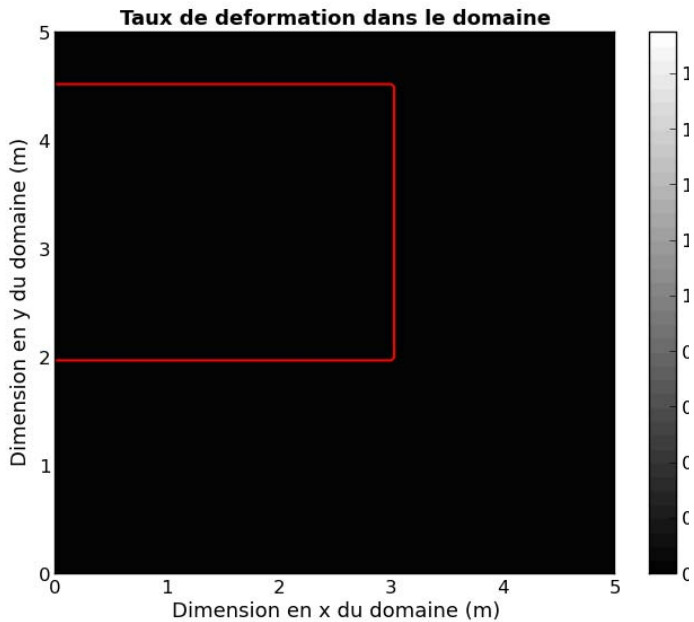

Figure 2. Déformée de la poutre à $t=3 \mathrm{~s}$ avec $\mu=5 \times 10^{7} \mathrm{Pas}$.

Le graphique ci-dessous (figure 3) présente les résultats de l'étude paramétrique menée sur l'influence de la valeur de la viscosité dynamique sur l'efficacité de la rigidification. La "zone de rigidification" (i.e., $\mu \in\left[10^{7} \mathrm{~Pa} \mathrm{~s} ; 10^{11} \mathrm{~Pa} \mathrm{~s}\right]$ ) représente la zone dans laquelle la rigidification est effective. Si l'on se trouve dans la zone "obstacle", la pénalisation est tellement forte que la partie de fluide solidifié se comporte tel un obstacle immobile (i.e., $u=0$ partout dans la zone). Enfin, pour des valeurs de $\mu$ trop élevées, le calcul diverge carrément. La taille des points du graphique sont représentatifs du temps de calcul de chaque simulation réalisée. On s'aperçoit qu'il existe une valeur optimale de $\mu$ (i.e., $\mu \sim 5 \times 10^{7} \mathrm{~Pa} \mathrm{~s}$ ) permettant à la fois une rigidification acceptable et un temps de calcul convenable.

On observe également une décroissance logarithmique de la valeur de $C_{\text {tot }}$ lorsque l'on fait augmenter la valeur de la viscosité dynamique $\mu$.

Cette méthode de pénalisation a été ensuite appliquée au cas de la chute libre d'une sphère dans un fluide visqueux (résultats non présentés dans cet article). En comparant nos simulations à des résultats expérimentaux, nous avons pu montrer que le modèle pouvait reproduire avec précision les efforts du fluide environnant sur le solide pour de faibles Reynolds. Par la suite nous contrôlerons ce phénomène à Reynolds élevés. 


\section{XIII ${ }^{\text {èmes }}$ Journées Nationales Génie Côtier - Génie Civil \\ Dunkerque, 2-4 juillet 2014}

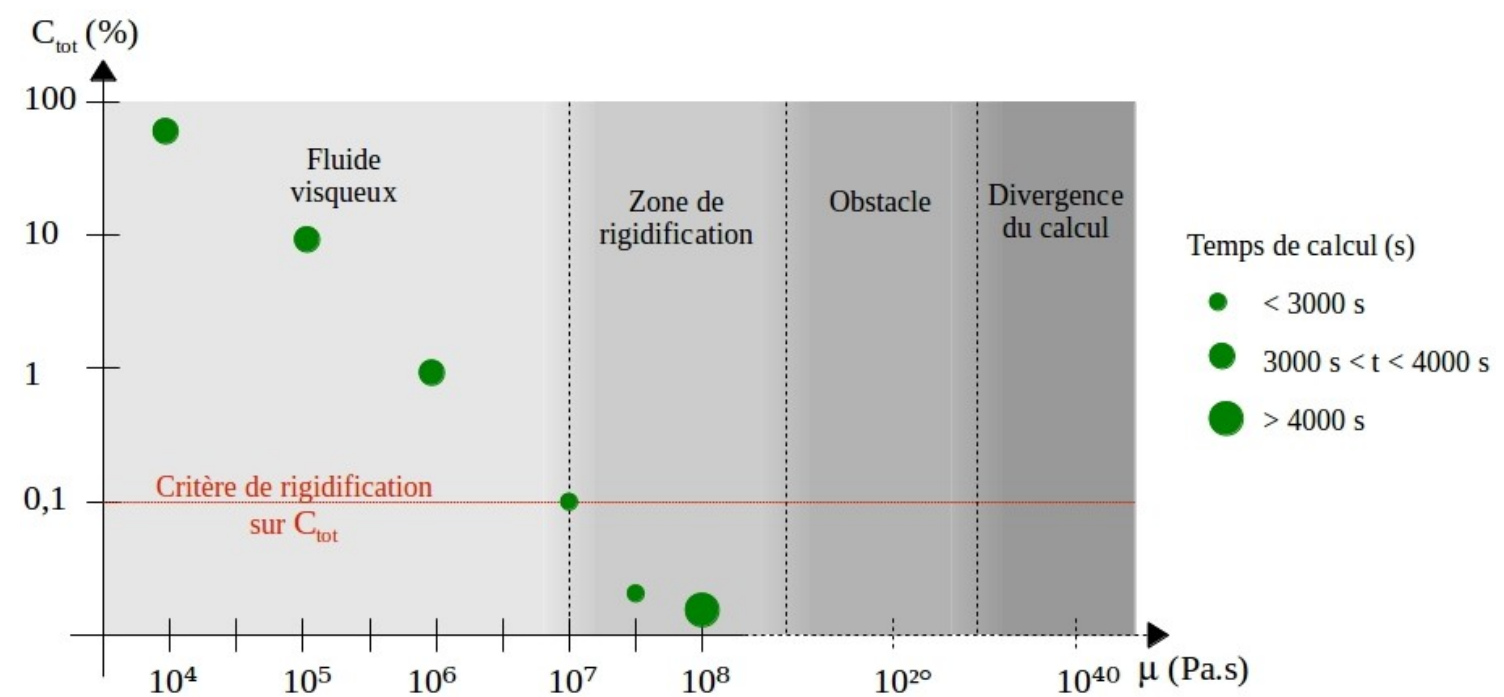

Figure 3. Évolution du critère de rigidification $C_{\text {tot }}$ en fonction de la viscosité dynamique $\mu$.

\subsection{Oscillateur amorti}

Nous avons ensuite testé notre approche dans le cas d'un solide pouvant se déplacer uniquement en translation et soumis à des efforts extérieurs de rappel (raideur $k$ ) et d'amortissement (coefficient $c$ ). L'objectif est d'appliquer par la suite ce type de modèle pour l'étude d'un système houlomoteur fonctionnant comme un batteur vertical inversé. Dans un premier temps, nous cherchons à tester la capacité du modèle à reproduire la vibration d'un oscillateur amorti.

Le problème étudié ici fait intervenir un bloc de forme carré de 1m de côté et de masse volumique $1000 \mathrm{~kg} \mathrm{~m}^{-3}$, posé sur le sol (sans frottement) soumis à une force volumique de rappel et à un amortissement dont nous ferons varier les valeurs. Le domaine de calcul est de largeur $9 \mathrm{~m}$ et de hauteur $3 \mathrm{~m}$. À l'état initial le bloc est excentré de 2,5 m par rapport à sa position d'équilibre. On étudie ensuite l'oscillation de celui-ci autour de cette position d'équilibre. Les conditions limites du domaine sont de type glissement. Les forces agissant sur le bloc sont la force de rappel, l'amortissement et les forces de surface générées par le fluide environnant, ici de l'air (i.e., $\rho \sim 1,18 \mathrm{~kg} \mathrm{~m}^{-3}$, $\mu \sim 1,85 \times 10^{-5}$ Pa s).

Nous pouvons supposer qu'un tel oscillateur est régi par l'équation différentielle (5), présentée ci-dessous, relative à un oscillateur masse-ressort-amortisseur à un degré de liberté, amorti par frottement fluide et soumis à un fluide environnant :

$\left(m+m_{a}\right) \ddot{x}+c \dot{x}+\frac{1}{2} \rho_{\text {fluide }} S_{\text {frontale }} C_{x} \dot{x}^{2}+k x=0$

où $x$ est le déplacement du bloc par rapport à sa position d'équilibre $x_{0}$. En première approximation on suppose que les effets du fluide environnant peuvent être décrits par une masse ajoutée $m_{a}$ et un coefficient de traînée $C_{x}$. Ceci est une approximation assez 


\section{Thème 5 - Énergies et ressources marines}

grossière car les allers et retours du solide perturbe graduellement l'écoulement de l'air, ce qui est assez différent d'un problème classique de traînée. La méthode de RungeKutta nous permet de trouver une solution numérique approchée de l'équation (5). Notons que sans le terme de traînée, une solution analytique peut être dérivée. En se basant sur cette solution analytique, en fonction des valeurs des constantes de raideur $k$ et d'amortissement $c$, nous allons pouvoir rencontrer quatre régimes différents (i.e., périodique, pseudo-périodique, critique et apériodique).

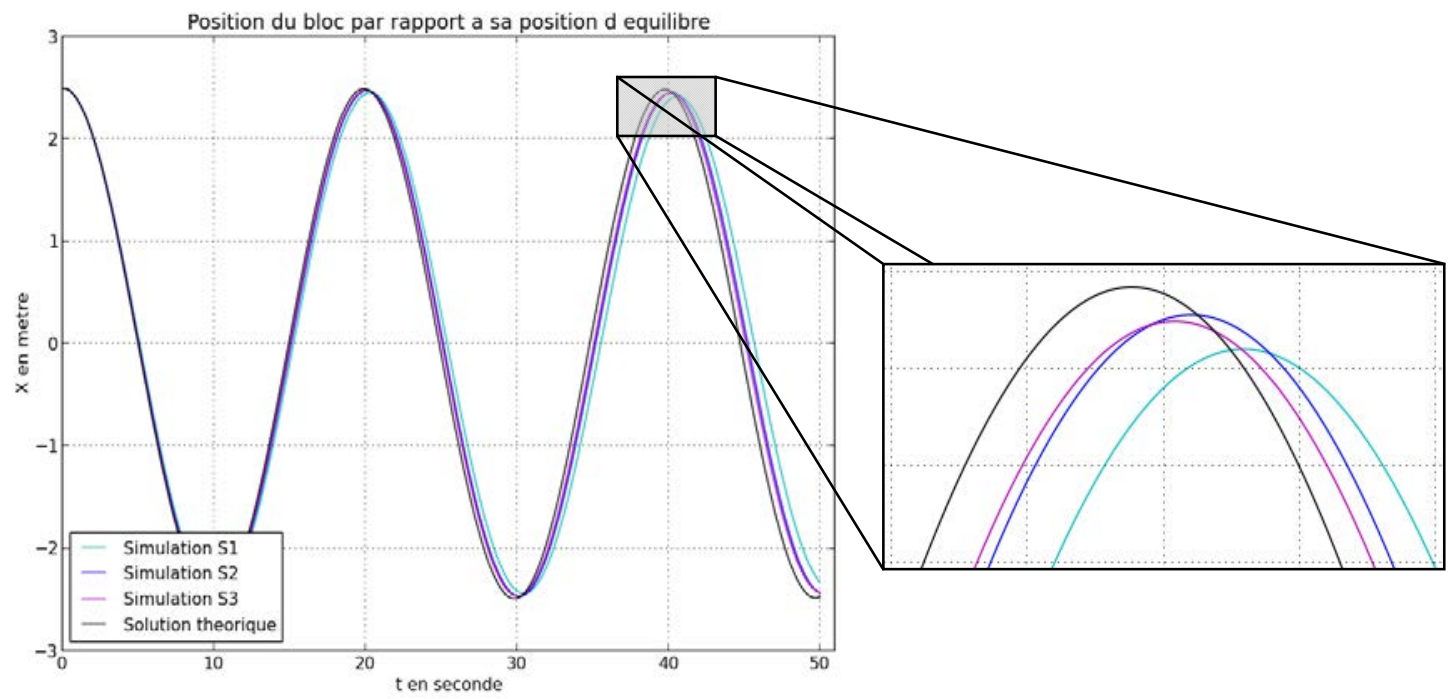

Figure 4. Réponse du système en fonction du maillage ; les simulations S1, S2 et S3 correspondant respectivement à des maillages $\Delta r e f, \Delta r e f / 2$ et $\Delta r e f / 3$.

Dans un premier temps nous avons choisi une solution dite périodique et nous avons étudié la sensibilité au maillage de la réponse numérique. Nous avons fixé un maillage de référence $\Delta_{r e f}=\delta x=\delta y=0,06 \mathrm{~m}$ puis nous l'avons fait évolué tout en étudiant la précision de notre réponse.

Les résultats de chacune de nos simulations numériques sont comparés à la solution analytique approchée par Runge-Kutta. La figure 4 ci-dessus montre l'évolution temporelle de la position du centre de gravité du bloc pour chacune des simulations effectuées ainsi que la solution théorique du système.

Ces résultats montrent que la solution numérique se rapproche de la solution théorique lorsque le maillage devient plus fin : le déphasage entre la solution numérique et la solution analytique diminue significativement et l'amplitude se rapproche de l'amplitude théorique.

Nous avons ensuite modifié les paramètres $c$ et $k$ afin d'étudier la capacité de notre méthode à simuler des systèmes à régime pseudo-périodique et apériodique (figure 5). L'erreur entre la solution théorique et numérique est relativement faible pour chacune des simulations, nous avons ainsi validé notre approche dans le cas d'un solide pouvant 


\section{XIII ${ }^{\text {èmes }}$ Journées Nationales Génie Côtier - Génie Civil \\ Dunkerque, 2-4 juillet 2014}

se déplacer uniquement en translation et soumis à des efforts extérieurs de rappel (raideur $k$ ) et d'amortissement (coefficient $c$ ).
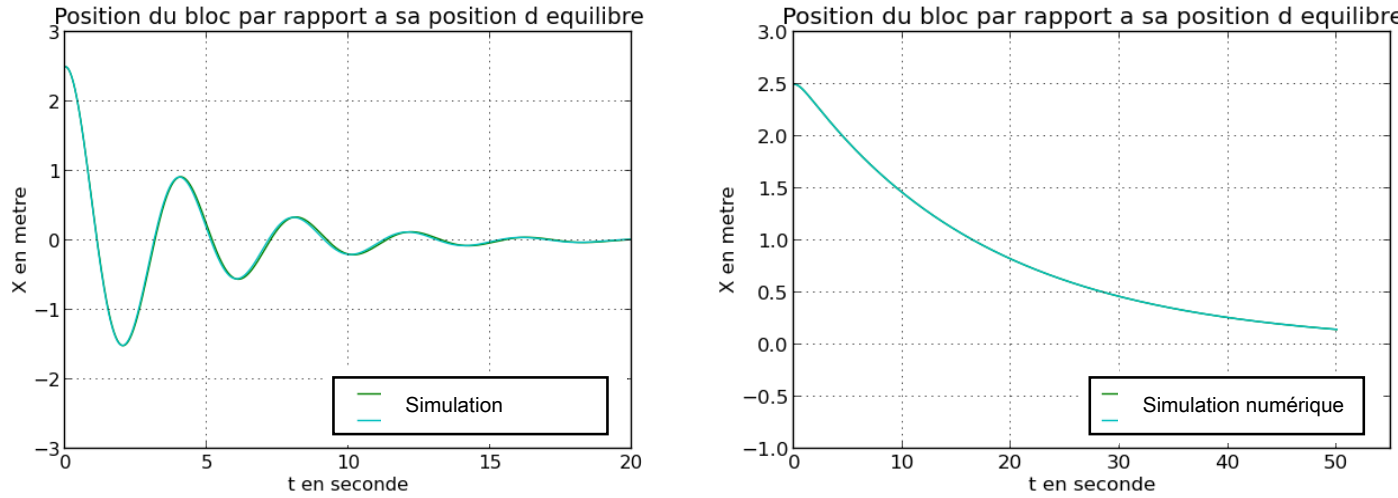

Figure 5. Comparaison entre les réponses numériques et analytiques de systèmes à régime pseudo-périodique (à gauche) et apériodique (à droite).

Pour terminer, nous envisageons de comparer une des simulations précédentes avec le même système oscillatoire masse-ressort-amortisseur en modifiant le fluide environnant. Cela nous permettra de mettre en avant les efforts de traînée et la prise en compte des masses d'eau (d'air) ajoutées pour des Reynolds élevés.

\section{Conclusion}

Nous avons développé une méthode originale permettant de modéliser l'interaction fluide/structure pour certains types de mouvement en utilisant uniquement un modèle Navier-Stokes. Elle permet en particulier de modéliser correctement un oscillateur à un degré de liberté amorti par un fluide environnant. Deux applications directes sont à l'étude :

- Un houlomoteur de type batteur inversé en translation dans le cadre du projet national EMACOP : l'objectif de ce projet est d'étudier le rendement et la survivabilité d'un tel système.

- Un système d'atténuation du ballottement dans le cadre d'une collaboration ECOSCONICYT entre le SIAME (Université de Pau et de Pays de l'Adour) et le DIMEC (Universidad de Santiago de Chile) : réalisation d'une série d'expérience sur table vibrante.

Afin d'enrichir la méthode en cours de développement, l'étude d'un oscillateur en rotation est en cours; les résultats préliminaires sont encourageants mais mettent également en évidence des problèmes techniques qu'il conviendra de régler dans l'avenir. 
Thème 5 - Énergies et ressources marines

\section{Remerciements}

Ce travail a reçu le soutien financier du programme national EMACOP. Il est également effectué dans le cadre du programme d'échange ECOS-SUD (et CONICYT) avec le Chili.

\section{Références bibliographiques}

ABADIE S., MORICHON D., GRILLI S., GLOCKNER S. (2010). Numerical Simulation of waves generated by landslides using a multiple-fluid Navier-Stokes model. Coastal Engineering, Vol. 57, pp 779-794. http://dx.doi.org/10.1016/j.coastaleng.2010.03.003 FORTIN M., GLOWINSKI R. (1982). Méthodes de lagrangien augmenté. application à la résolution numérique de problèmes aux limites. Dunod, Paris.

GLOWINSKI R., PANA T.-W., HESLAB T.I., JOSEPH D.D. (1999). A distributed Lagrange multiplier/fctitious domain method for particulate fows. International Journal of Multiphase Flow, Vol. 25, pp 755-794. http://dx.doi.org/10.1016/S0301-9322(98)00048-2

MOKRANI C. (2012). Impacts de vagues déferlantes sur un obstacle vertical. Modèle théorique et estimation numérique des pics de pression, Thèse de l'Université de Pau et des Pays de l'Adour, 13 février 2012, 187 p.

RITZ J.B., CALTAGIRONE J.P. (1999). A numerical continuous model for the hydrodynamics of fluid particule systems. International journal for numerical methods in fluids, Vol. 30, pp 1067-1090. http://dx.doi.org/10.1002/(SICI)1097-0363(19990830)30:8<1067::AIDFLD881>3.0.CO;2-6

PATANKAR S. (1990). Numerical heat transfert and fluid flow. Hemisphere publishing Corporation, NewYork.

WILLIAM J.R., DOUGLAS B. (1998). Kothey Reconstructing Volume Tracking. Journal of Computational Physics, Vol. 141, pp 112-152. http://dx.doi.org/10.1006//cph.1998.5906 